\title{
A study of the construction times of the ancient cities in Ganjia Basin, Gansu Province, China
}

\author{
XIA Huan ${ }^{1},{ }^{*}$ ZHANG Dongju', WANG Qiang ${ }^{1}$, WU Duo ${ }^{1}$, DUAN Yanwu ${ }^{1}$, \\ CHEN Fahu ${ }^{2,3}$
}

1. Key Laboratory of Western China's Environmental Systems (Ministry of Education), College of Earth Environmental Sciences, Lanzhou University, Lanzhou 730000, China;

2. Key Laboratory of Alpine Ecology, Institute of Tibetan Plateau Research, CAS, Beijing 100101, China;

3. Center for Excellence in Tibetan Plateau Earth Sciences, CAS, Beijing 100101, China

\begin{abstract}
The Ganjia Basin in Xiahe County, Gansu Province, China, is located on the northeastern edge of the Tibetan Plateau. The area lies in the transitional zone between the Plateau ethnic groups dominated by ancestral Tibetans, and the Central Plains dynasties dominated by ancestral Han in history. The Ganjia Basin is therefore well suited to studying the history of the intermixing of the Han and Tibetan peoples. In this study, we collected samples of organic materials from two ancient cities, Bajiaocheng (BJC) and Sirougucheng (SRGC), in the Ganjia Basin, which were used for radiocarbon dating. Our aims were to determine the construction times and function of the cities, based on the radiocarbon ages, Bayesian age modeling, and the analysis of relevant historical records. The results are used to discuss the role of the Ganjia Basin in the conflicts between and the integration of the two ethnic groups during the historical period. We conclude that BJC was established during 663-732 CE (Common Era); its early occupation history can be divided into an initial establishment phase (670-780 CE) and a phase of regeneration (880-1030 CE). SRGC was probably built at the time of the transition between the Song and Yuan dynasties ( 1271 CE). Combining the dating results with an analysis of the architectural styles and historical records, we conclude that BJC was originally built by the Tubo Kingdom as a military stronghold, and its major functions were military, economic and religious, and to support the major eastward expansion of the Tubo. SRGC was most likely a temple-focused city intended to promote Tibetan Buddhism which was a major political and religious leadership for the Tibetans at that time, reflecting changes in the status of different religions in the Ganjia Basin. In addition, the intermixing and blending of the Han and Tibetan groups, reflected by the human activities in the Ganjia Basin, was an important demographic and cultural foundation for the formation of the culturally-diverse but spiritually-united modern Chinese people.
\end{abstract}

Keywords: Ganjia Basin; radiocarbon dating; Bajiaocheng; Sirougucheng; ethnic amalgamation; Tubo

Received: 2020-04-25 Accepted: 2020-06-26

Foundation: National Key R\&D Program of China, No.2018YFA0606400; Strategic Pilot Science and Technology of the Chinese Academy of Sciences Project, No.XDA20040000; Fundamental Research Funds for the Central Universities, No.lzujbky-2016-279

Author: Xia Huan (1994-), PhD Candidate, specialized in environmental archeology. E-mail: xiah2012@1zu.edu.cn

"Corresponding author: Zhang Dongju (1981-), Professor, specialized in environmental archeology.

E-mail: djzhang@1zu.edu.cn 


\section{Introduction}

The Chinese mainland, with the geographical barriers of ocean in the south, the Mongolian Plateau in the north, the Pamir Plateau and the Altai Mountains in the northwest, and the Tibetan Plateau in the southwest, provided a relatively independent space for the survival and growth of the Chinese people for thousands of years (Fei, 1999). Within this semi-closed environment, rich and varied cultures and multi-ethnic groups have developed within the diverse geographical units (An, 2007). Although the relationships between these groups are complex, an integration of the Chinese nation eventually occurred in the course of their interactions and communications (Fei, 1999). In particular, the long-standing connections between the plateau ethnic groups, which are mainly composed of generations of Tibetans occupying the Tibetan Plateau, and the low-altitude ethnic groups which are dominantly composed of the Han, to a certain extent promoted the formation of the modern Chinese nation. According to archaeological, genetic and linguistic studies (Chen et al., 2015a; Li et al., 2019; Zhang et al., 2019), modern Tibetans principally originated in northern China and they have a close relationship with the spread of Neolithic millet farmers associated with the Yangshao Culture and Majiayao Culture in the middle Yellow River. Furthermore, the cultural and political conflicts and integrations between the peoples of the Plateau and the low-altitude regions in the historical period further deepened the relationship between the Han and the Tibetans (Wang, 2006; Cui and Ma, 2009; Shi, 2016). Therefore, the study of the history of the blending of the ancestral Han and Tibetans can help us to understand the process of the establishment of the modern Chinese nation.

The Ganjia Basin is located in the northeast margin of the Tibetan Plateau, on the boundary of Qinghai and Gansu provinces in China, and it is considered to be one of the earliest pathways for prehistoric human migration to the Plateau. The earliest evidence of human activity on the Tibetan Plateau, the Xiahe mandible, was discovered in the Ganjia Basin (Chen et al., 2019). Over a long period, the basin was also located in the transitional region between the Central Plains dynasties and the Plateau ethnic groups. As an important center supporting the eastward expansion of the Plateau ethnic groups, the Ganjia Basin was a significant military stronghold for the Central Plains dynasties in their competition with the Qiang ( 1600 BCE-600 CE), Tuyuhun (313-663 CE), Tubo (633-842 CE), Tsongkha (1008-1099 CE) and other Plateau ethnic groups or regimes. Therefore, the basin is well suited for studying the history of the interaction and integration of the ancestral Han and Tibetans. So far, three ancient cities-Bajiaocheng (BJC), Sirougucheng (SRGC) and Gadaikalacheng (GDKLC)-have been discovered during archaeological surveys in the region. Although the ages of these ancient cities have been surmised from the study of historical documents and archaeological remains (Li, 1994; Yi, 1994; Guo, 2010; Jiucaoxi, 2010; Zhou and Fan, 2010), their construction times and functions are still highly debated, especially given the absence of reliable dating results and the limitations of historical records (Tang and $\mathrm{Li}, 2017)$. Radiocarbon dating, as a reliable dating method with high precision and wide applicability, has been successfully used in the dating of many ancient cities (Dong et al., 2016; Guo et al., 2018; Liu et al., 2019). In the present study, samples of organic materials were collected from BJC and SRGC, whose walls are still preserved in the Ganjia Basin, in order to determine the history of their construction and hence the ages of the cities. Addi- 
tional aims were to determine the functions of the cities based on an integration of the radiocarbon dating results and historical documentary evidence, and the role of the Ganjia Ba$\sin$ in the conflicts between and integration of the ancestral Han and Tibetan peoples.

\section{Study area}

The Ganjia Basin, with an average elevation of $2900-4636 \mathrm{~m}$, is located $\sim 25 \mathrm{~km}$ north of Xiahe County in Gansu Province. The terrain is inclined from northwest to southeast, and the highest point is the main peak of Dalijiashan Mountain, the northern offset of the Qilian Mountains. The basin is penetrated by the Yangqu River, the major tributary of the Xiahe River, which is mainly supplied by groundwater and meltwater (Figure 1). From the Ganjia Basin, there is a routeway passing through upstream along the Yangqu River and crossing a pass in Dalijia Mountain to Qinghai Province. A second routeway exists downstream along the Yangqu River and Daxia River to Linxia Hui Autonomous Prefecture in Gansu Province. The climate in the basin is influenced mainly by the East Asian monsoon (Chen et al., 2015b). The mean annual precipitation ranges from 346 to $360 \mathrm{~mm}$, and the mean annual temperature is $1{ }^{\circ} \mathrm{C}$, and with only 120 frost-free days per year (http://www.xiahe.gov.cn/ qtpagedata/articaldisp $187 . \mathrm{html}$ ). The vegetation in the basin is mainly alpine meadow, and the land use is mainly animal husbandry supplemented by cultivation.

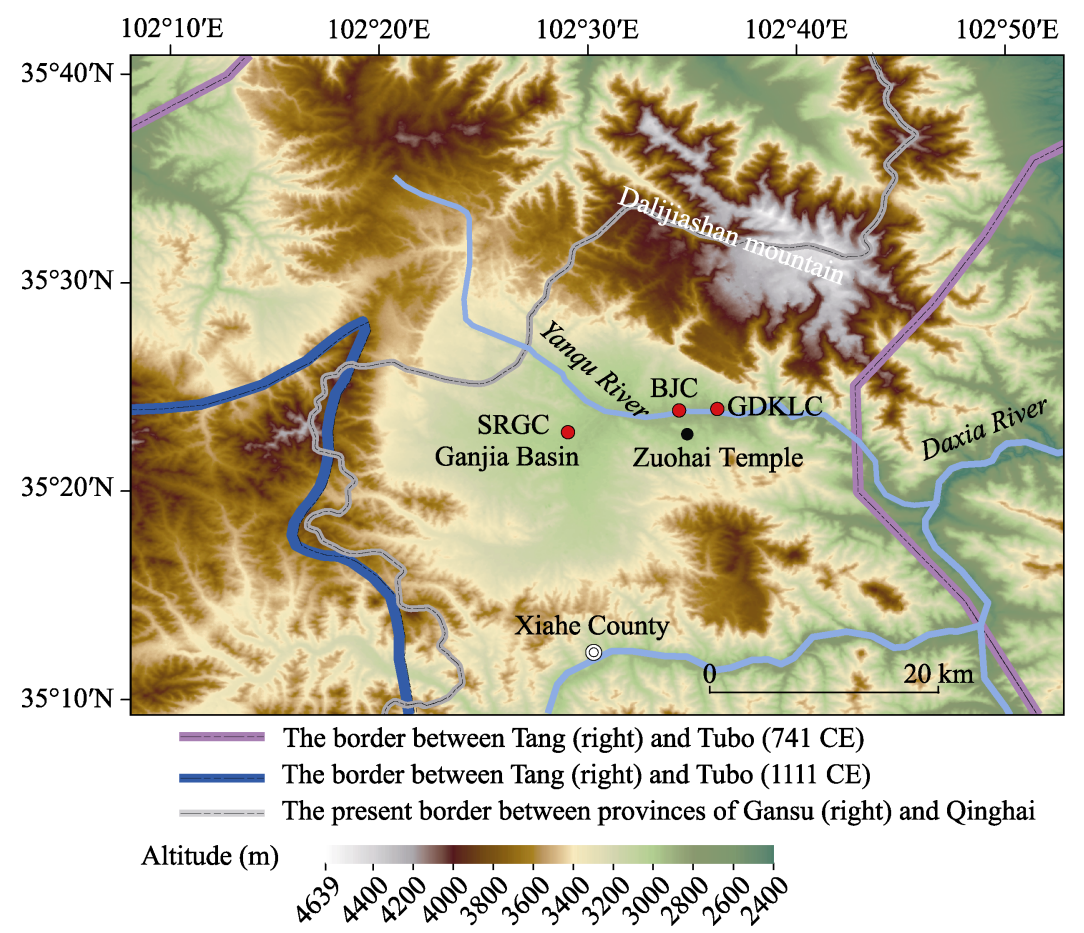

Figure 1 Location of ancient cities in the Ganjia Basin. The historical borders are based on Tang (1982).

Three ancient cities (BJC, SRGC and GDKLC) are distributed in the Ganjia Basin, but ancient walls are only preserved in BJC and SRGC (Figure 1). BJC, named after its eight corners and at an altitude of $2940 \mathrm{~m}$, is located on a platform at the confluence of the Yangqu River and its tributary the Jiangla gully (Figure 1). Its Tibetan name is related to the 
“‡" symbol of the Bon religion, which means "eternal and solid"; the shape of the city resembles this symbol, which may reflect the belief system of the Bon religion practiced by those who constructed the city (Zhou and Fan, 2010). The total length of the existing walls is $2193.4 \mathrm{~m}$, with a maximum height of $10 \mathrm{~m}$ and covering an area of $0.2 \mathrm{~km}^{2}$. Moats and a bulwark are present outside the existing walls, and an additional building complex is located outside the south gate. The walls were constructed using the technique of compressing soil between wooden frames. In previous archaeological excavations at BJC, pottery shards from Neolithic period, Tang (618-907 CE) and Song (960-1279) dynasties, as well as a large number of copper coins from Song Dynasty, were excavated (Li, 2012). Han Dynasty tombs are located in the uplands northwest of the city (Guo, 2010). According to its architectural features, geographical location, archaeological remains and historical records, BJC was regarded as Baishi County in the Han Dynasty (202 BCE-220 AD) (Yi, 1994); however, this conjecture was overturned because of no exact evidences. Based on historical records written in both Chinese and Tibetan characters, Zhou and Fan (2010) concluded that BJC was constructed during the Northern Song Dynasty (997-1065 CE) by Gusiluo, who was a descendant of the Tubo royal family and established the Tsongkha regime that ruled northeastern Tibetan Plateau (1009-1099 CE).

SRGC, at an altitude of $3000 \mathrm{~m}$, is located in the central area of the Ganjia Basin, on the platform to the northwest of Sirou village, near the confluence of the tributaries of the upper reaches of the Yangqu River (Figure 1). Although most of the inner and outer walls have been destroyed, the planar structure of the walls is still visible. A partially-preserved building is present in the center of the inner city. In plan view, the SRCG is an irregular rectangle with an area of $40,000 \mathrm{~m}^{2}$. The construction technology is the same as that used for BJC. Surveying and mapping by $\mathrm{Li}$ (1994) indicated that the construction style followed that of traditional Chinese palace architecture. Abundant pottery shards with Plateau ethnic characteristics and large-scale plinths with honeysuckle and lotus motifs have been excavated within the city. Combined with the archaeological remains and historical records, Li (1994) concluded that SRGC was constructed during the Northern Song Dynasty (960-1127 CE) by the Tsongkha and was named "Yigong city".

\section{Materials and methods}

During fieldwork in 2014 we collected samples of 39 plant remains from BJC and SRGC, including 38 samples from walls and 1 sample from the central building in SRGC. In order to avoid damage to the walls, only plant materials that were exposed on the exterior were collected; however, this limited our ability to understand the overall structure of the walls. The lower part of the walls was more affected by human activities and natural processes (such as water erosion and weathering), with the result that more of the interior materials of the walls were exposed (Figure 2). Dates from samples near the base of walls are closer to the date of initial construction, and therefore in order to accurately reflect the construction time, most of the samples were collected from the bases. We speculate that BJC may have been repaired numerous times during different historical periods, based on its good state of preservation. Therefore, several samples were also collected at the bottom of the wall (1-2 m above the base) to reflect multi-time repairment of it. In order to reduce or minimize the in- 
fluence of the "old wood effect" (Schiffer, 1986; Zilhão, 2001; Yizhaq et al., 2005; Nowak et al., 2017), the collected samples comprised mainly twigs and herbaceous plant material, while wood samples were collected from the outer part of the frame splint. Ten samples were selected for radiocarbon dating (Figure 2); they include charcoal (SRGC1401, SRGC1404), wood (BJC1401, BJC1408 and BJC1420), twigs and herbaceous plant material (BJC1412, BJC1415, BJC1422, BJC1429 and BJC1431) (Figure 2). Three samples (BJC1401, BJC1408 and BJC1431) were collected from $\sim 1-2 \mathrm{~m}$ above the base of walls, and the other samples were all from the bases of walls.

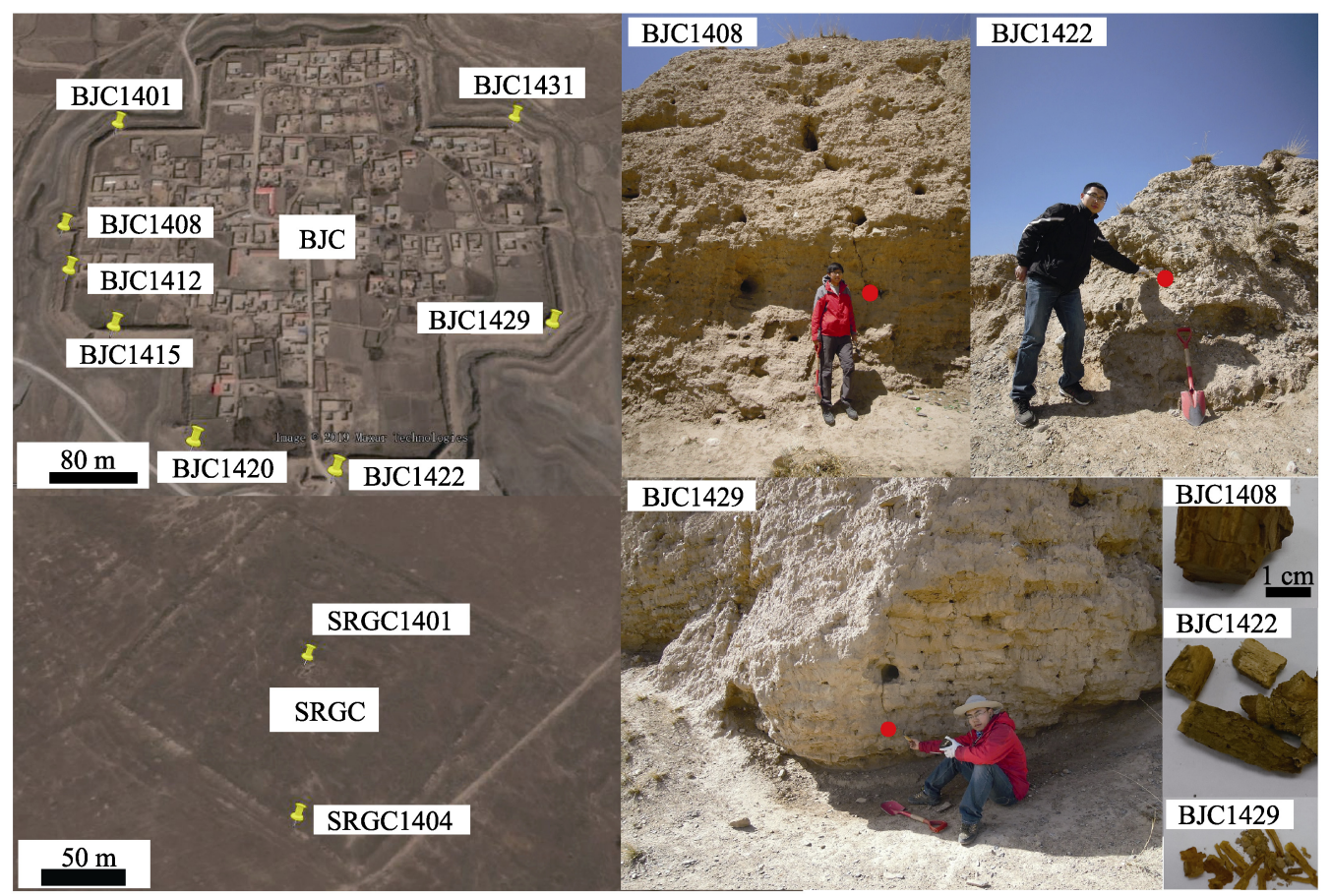

Figure 2 Sampling locations in the ancient cities of BJC and SRGC and photographs of samples

The samples were pretreated at the MOE Key Laboratory of Western China's Environmental System in Lanzhou University using the procedures of Liu et al. (2019), and AMS ${ }^{14} \mathrm{C}$ measurements were made at Peking University. All of the dates were calibrated to calendar ages using the IntCal13 calibration curve (Reimer et al., 2013) in OxCal v4.3 and expressed as cal BCE/CE (Before Common Era/Common Era). Bayesian modelling of radiocarbon ages was carried out using the built-in "phase" and "sum" models in OxCal v.4.3. In order to evaluate the "old wood effect" of the dated samples, species identification was carried out on samples of wood and charcoal.

\section{Results}

The radiocarbon dating results are listed in Table 1. The range of all calibrated ages from BJC is $662-1147$ cal CE (95.4\% range), corresponding to the period from the Tang Dynasty (618-907 CE) to the Northern Song Dynasty (960-1127 CE). The oldest date is from a twig (BJC1422) with a maximum diameter of $3 \mathrm{~mm}$ (Figure 2), almost entirely excluding the influence of "old wood effect". The dates of wood samples from BJC were no older than those 
of other samples, which may be related to the fact that the exterior of the wood was sampled. As expected, the ages of samples collected from $\sim 1-2 \mathrm{~m}$ above the base of the walls (883-1147 cal CE, 95.4\% range) are younger than those of the samples from the base.

Table 1 Radiocarbon dates from the ancient cities in the Ganjia Basin

\begin{tabular}{llcccc}
\hline \multirow{2}{*}{ Number } & Dating material & ${ }^{14}$ C date (a BP) & \multicolumn{3}{c}{ Calibrated age (cal CE) } \\
\cline { 5 - 6 } & & & $1 \delta(68.2 \%)$ & $2 \delta(95.4 \%)$ & Median \\
\hline SRGC1401 & Charcoal (Picea) & $615 \pm 25$ & $1300-1394$ & $1295-1400$ & 1350 \\
SRGC1404 & Charcoal (Populus) & $740 \pm 25$ & $1263-1282$ & $1225-1290$ & 1271 \\
BJC1408 & Wood (Picea) & $1010 \pm 25$ & $995-1029$ & $981-1147$ & 1018 \\
BJC1401 & Wood (Picea) & $1105 \pm 50$ & $888-992$ & $777-1020$ & 931 \\
BJC1431 & Twigs and herbs & $1115 \pm 25$ & $895-971$ & $883-990$ & 935 \\
BJC1412 & Twigs and herbs & $1160 \pm 30$ & $778-944$ & $773-968$ & 870 \\
BJC1415 & Twigs and herbs & $1240 \pm 35$ & $689-861$ & $681-881$ & 765 \\
BJC1429 & Twigs and herbs & $1250 \pm 25$ & $690-774$ & $677-866$ & 736 \\
BJC1420 & Wood (Pinus) & $1285 \pm 25$ & $681-766$ & $668-770$ & 715 \\
BJC1422 & Twigs and herbs & $1300 \pm 25$ & $669-764$ & $662-769$ & 705 \\
\hline
\end{tabular}
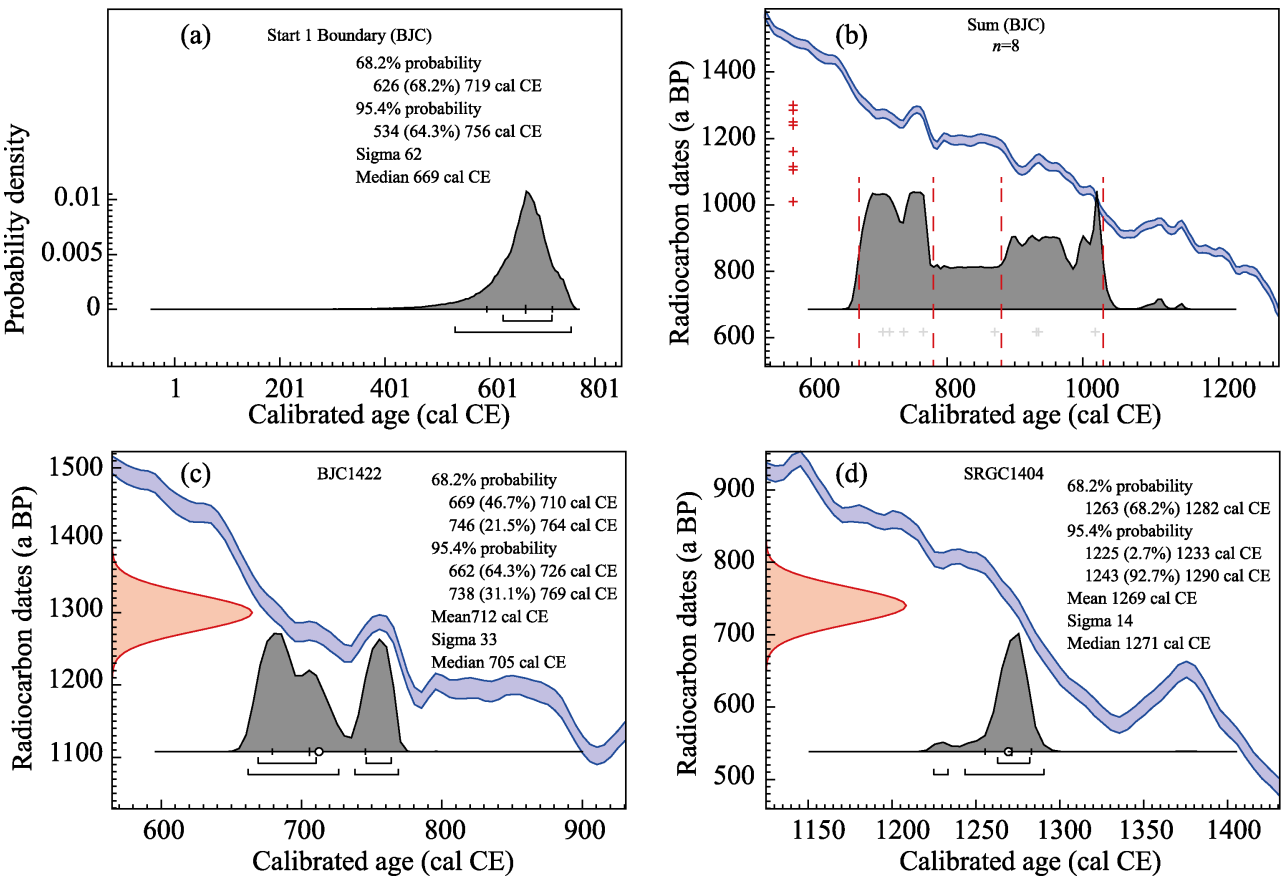

Figure 3 Dating results of the samples from ancient cities in the Ganjia Basin: (a. "phase" modeled age ranges for Bajiaocheng; $b$. summed probability distributions of eight radiocarbon dates from Bajiaocheng; c. calibrated radiocarbon dates for BJC1422; d. calibrated radiocarbon dates for SRGC1404)

The range of all of the dates from SRGC is 1225-1400 CE (95.4\% range), corresponding to the period from the Southern Song Dynasty (1127-1279 cal CE) to the Ming Dynasty (1368-1644 CE). The ages of the sample from the walls range from 1225-1290 CE, slightly older than age of the sample from the central building (1295-1400 cal CE), indicating that 
the two buildings were constructed at different times. The charcoal samples from SRGC were identified as Picea and Populus (Table 1), both of which are woody perennials, indicating that the dating results may be influenced by the "old wood effect".

The results of the "phase" and "sum" models for the dates from BJC are shown in Figures $3 \mathrm{a}$ and $3 \mathrm{~b}$. The range of the upper median age boundary is $669 \pm 63 \mathrm{cal} \mathrm{CE}$ based on the "phase" model (Figure 3a), and the "sum" model shows that the ages of BJC are mainly concentrated within two ranges: $670-780 \mathrm{cal} \mathrm{CE}$ and $880-1030 \mathrm{cal} \mathrm{CE}$. The median range of the sample (BJC1422) representing the earliest construction age of $\mathrm{BJC}$ is $705 \pm 33 \mathrm{cal} \mathrm{CE}$ (Figure 3c); the oldest sample from SRGC is SRGC1404, with the age median range of $1271 \pm 14$ cal CE (Figure 3d).

\section{Discussion}

\subsection{Construction history of the ancient cities in the Ganjia Basin}

In the early Tang Dynasty, the Ganjia Basin was under the jurisdiction of the Tuyuhun, an ancient kingdom in the northeastern Tibetan Plateau (284-670 CE), and was located on the border of the Tuyuhun and the Tang Dynasty. From 649 CE, following the death of Songtsen Gampo (the king and founder of the Tubo Kingdom), the Tubo Kingdom was ruled by the Gaer family, which ended the brief period of peaceful coexistence with the Tang Dynasty. The Tubo Kingdom than commenced a period of military expansion into the surrounding areas (Tian, 2016), eventually occupying the entire territory of Tuyuhun in $663 \mathrm{CE}(\mathrm{Pu}$, 2017). Due to its geographical advantages of connections with Hezhou (in modern Linxia county, Gansu province) in the Longyou area, and with Qinghai, that were respectively under the control of the Tang Dynasty and the Tubo, the Ganjia Basin became the center of military conflict between these two regimes in the northeastern margin of the Tibetan Plateau. Therefore, we hypothesize that Tubo established a military base centered in the Ganjia area by constructing BJC, and attempted to conquer the Central Plains regimes. This interpretation is consistent with historical records which indicate that the Tubo frequently invaded the Longyou area following the annexation of Tuyuhun (Wang, 2006). At the same time, the Bon religion spread into Tuyuhun with the expansion of the armed forces of the Tubo, which may explain why the architectural style of BJC exhibits a strong Bon cultural influence. The radiocarbon dates reported herein confirm this conjecture. The median age range of the upper limit in the "phase" model $(669 \pm 63$ cal CE) represents the earliest construction age of BJC, and combined with the historical records dating to the time when the Tubo occupied the Ganjia Basin, we can constrain the age range of the initial construction of BJC to 663-732 cal CE. Two prominent peaks in the "sum" model correspond to the major period of frequent restoration of BJC. The first peak (670-780 cal CE) represents the beginning of construction of BJC as a military stronghold and its initial repair, which is termed the initial establishment period; and the second peak (880-1030 cal CE) represents the repair history of BJC, which is termed the regeneration period.

Our conclusion regarding the earliest construction time of BJC differs from that of previous studies which suggested that it was constructed during the Western Han Dynasty (206 BCE-9 CE), the Northern and Southern Dynasties (386-589 CE), or the Northern Song 
Dynasty (960-1127 CE). The previous mainstream view was that BJC was built in $1028 \mathrm{CE}$ by Gusiluo (997-1065 CE), the king of Tsongkha. This view is based on the following Tibetan historical records. There was the BJC on the other side when the Zuohai Bon temple was built in $1028 \mathrm{CE}$ in the Ganjia Basin (Figure 1). However, there is no unambiguous record indicating that the construction of BJC was contemporaneous with that of the Zuohai Bon temple. The dating results presented herein indicate that $\mathrm{BJC}$ already existed at the time when the temple was built.

Sample SRGC1404, with the median age range of $1271 \pm 14 \mathrm{cal}$ CE and collected from one of the walls of SRGC, is more representative of its construction history than sample SRGC1401 from the central building (Figure 3d). In contrast to the samples collected from the BJC, the age of the sample may be older than the actual age of the construction of SRGC due to the "old wood effect" (Dong et al., 2014). Therefore, the construction age of SRGC should not have been earlier than $1271 \pm 14$ cal CE. Li (1994) considered that its architectural style indicates an age no earlier than the Sui and Tang dynasties and no later than the Ming and Qing dynasties, which associates it with the city of "Yigong" in the area, which was constructed at the same period. Clearly, however, the evidence to confirm this supposition is insufficient. According to Chinese historical records (Toqto, 2012), Gusilou was taken in $1008 \mathrm{CE}$ from Gaochang to the Hezhou area by Helangye, a merchant, and was subsequently installed in "Yigong" of Xiahe by the local leader, indicating that the city had existed previously. Combined with the dating results and the building scale of BJC and SRGC, it appears more likely that "Yigong" city is BJC. In around $1270 \mathrm{CE}$, the Sakya Tibetan Buddhist sect led by Drogön Chogyal Phagpa (the fifth leader of the sect) became the political and religious leader of the Tubo Kingdom. The large-scale plinths with honeysuckle and lotus motifs in the SRGC reflect those of Tibetan Buddhism (Li, 1994). Combined with the dating results, we assume that SRGC was probably a city centered on the Tibetan Buddhist temple and that its abandonment may have been related to the decline of the Sakya sect.

\subsection{The functions of the ancient cities in the Ganjia Basin}

The layout of BJC indicates its important military function. The octagonal shape interrupts the square shape of the former city. Each corner of BJC is within the effective range of a bow and crossbow which facilitated its defense. Combined with the distinctive geographical location of the Ganjia Basin, we infer that BJC was not only an important stronghold and gathering area for the Tubo to engage in military operations in the Longyou area, but it was also an important fortress which could be defended against the armies of the Tang Dynasty. The Longyou area was an important defense line for the Tang Dynasty to defend its capital city of Chang'an against the Tubo. After the Tubo occupied Tuyuhun, and especially after Yang Ju, the governor of Shanzhou (the territory includes the modern cities of Ledu, Xining and Huangzhong in Qinghai), ceded the land of Jiuqu (the area of the major bend of the Yellow River to the southeast of Qinghai) to Tubo in 712 CE (Liu, 1975; Wang, 2006). The wars between the Tang Dynasty and the Tubo Kingdom in the Longyou area became more frequent, which corresponded to the initial construction of BJC as indicated by the "sum" model (670-755 CE), reflecting the frequent maintenance and restoration of BJC during these wars. After the outbreak of the "An-Shi Rebellion" (755-763 CE), the Tubo Kingdom 
occupied the Longyou area (Liu, 1975). The Ganjia Basin was no longer the frontline of the wars between the Tang and Tubo and it lost its strategic significance. This period corresponds to the relatively uniform interval between the two high amplitude peaks in the "sum" model (Figure 3). In $842 \mathrm{CE}$, Langdarma, the last emperor of the Tubo Kingdom, was assassinated by a Buddhist monk, which was followed by the Era of Fragmentation in the Tubo Kingdom, which lasted for nearly 400 years. During this period, the Tang Dynasty was briefly revived under the rule of Emperor Xuanzong II, the 19th Tang emperor. Therefore, from 848 to $851 \mathrm{CE}$, the Longyou area, occupied by the Tubo for nearly 100 years, was returned to the Tang Dynasty as a result of wars or the direct surrender by the regional warlords of the Tubo (Liu, 1975; Ouyang et al., 1975; Sima, 2016; Wang, 2006). Therefore, the Ganjia Basin once again became the border area between the Tang Dynasty and the Tubo tribes, and BJC again played an important role in military confrontation, which corresponds to the regeneration period reflected in the "sum" model (Figure $3 b$ ). The weakening of the military capability of the Tubo tribes also resulted in BJC no longer being a stronghold and launching area for attacks in Longyou; rather, it became a fortress for resisting the Central Plains dynasties. At the beginning of the 11th century, the Northern Song Dynasty supported the regime of Tsongkha, the Tubo tribes in Gansu and Qinghai. Both Gusiluo and his descendants were appointed officials by the Song Dynasty (Toqto, 2012). The amicable relations between the Song and the Tsongkha may be the main reason that BJC was no longer repaired frequently as a military stronghold. However, we cannot exclude the possibility that restoration after the Northern Song Dynasty has not been revealed because of the limitations of our sampling program, which was concentrated in the middle and lower parts of the walls.

Historical records show that the Ganjia Basin was not only a military stronghold but also an economic center of the region. It had abundant water and grazing resources for the nomadic Qiang people (Zhou and Fan, 2010). During the reign of the Tubo Kingdom (663-842 $\mathrm{CE}$ ), animal husbandry was an important economic base for its military expansion (Wang, 2006), and the growth of the area used for animal husbandry was a state policy pursued by the ruling class (Wei, 2013). Related to the expansionist strategy of the integrated military-civil leadership of the Tubo army (Ouyang et al., 1975), it can be inferred that the Tubo Kingdom would have taken advantage of the ideal geographical location and favorable environment of BJC to actively pursue animal husbandry and cultivation. Economic production units relying on military organization would have been formed which supported the expansion of Tubo to the Longyou area. During the Tsongkha period (997-1099 CE), there was a well-developed arable and pastoral economy in the Ganjia Basin. Contemporary Tibetan literature shows that there were thousands of farmers and herdsmen near the Zuohai Bon temple at the time of its construction in $1028 \mathrm{CE}$ (Zhou and Fan, 2010). Subsequently, during the period of the Northern Song Dynasty (1103-1127 CE) (Toqto, 2012; Nie and Hou, 2015), the government carried out a policy of reclaiming wasteland in order to sustain its military forces (Liu et al., 2014). Therefore, the large-scale abandonment of terraces on the flat land, hills and platforms in the Ganjia Basin may have occurred as a result of this active land reclamation policy (Zhou and Fan, 2010). Given the limitations of the altitude $(>3,000$ $\mathrm{m})$, it can be assumed that mainly cold-tolerant grains, especially upland barley and wheat, were cultivated (Liu, 1975; Chen et al., 2015a). This period, corresponding to the Medieval Climate Anomaly (MCA), was the longest sustained warm period in China during the past 
2,000 years (Ge et al., 2013; Zhang et al., 2019). Both stalagmite oxygen isotope records from caves in China and a pollen-based quantitative precipitation reconstruction from Gonghai Lake indicate a high intensity of the East Asian summer monsoon at this time (Zhang et al., 2008; Chen et al., 2015b). Therefore, the favorable climate, especially the relatively high precipitation, facilitated the implementation of the land reclamation policy. Overall, this resulted in the transformation of the economy of the Ganjia Basin from animal husbandry to cultivation. However, in 1742 CE, Ortai, a high official in the Qing Dynasty, wrote a memorandum to the Emperor Qianlong, the 6th Emperor of the Qing Dynasty (1711-1799 CE), stating that the lands in the Ganjia Basin were unsuitable for cultivation. This reflects the previous abandonment of cultivated areas in the Ganjia region, which may have been related to the occupation of nomadic groups who did not carry out appropriate land management policies following the Northern Song Dynasty, when the climate became colder after the MCA (Ge et al., 2013; Zheng et al., 2019) and the Asian monsoon and East Asian summer monsoon weakened (Zhang et al., 2008; Chen et al., 2015b). Finally, the local economy reverted once again to animal husbandry which continues to the present day.

The ancient cities of BJC and SRGC, which represent different religious orders, also reflect changes in the power relationships between religious groups in the Ganjia Basin. The Bon religion (500 BCE-625 CE), originating in Shangshung, an ancient kingdom on the Plateau, was introduced into the Tubo Kingdom when it was still the Yalong tribe. Its forces were strong enough to threaten royal power during the period when the Plateau was ruled by Drigum Tsenpo ( 146-123 BC), a king of Tibet in Tibetan mythology. However, religious and royal power remained in a relative balanced state after the failure of Drigum Tsenpo's effort to destroy the Bon religion (Cai, 2006). Although Songtsen Gampo introduced Buddhism to the Tubo Kingdom during his reign (629-650 CE), Bon still had an advantage during the subsequent five generations of the kings' reign due to its deep-rooted influence. During military expeditions, there were always Bon priests present who conducted important sacrificial rituals in order to solicit the assistance of the gods. A large number of Tibetan documents were found in Dangchang in Gansu Province, which recorded a battle between the Tang army and the practitioners of Bon (Yishi Wangmo and Dorji Tserin, 2015). The architectural style of BJC also reflected the far-reaching influence of Bon during this period. In the middle of the 8th century, Trisong Detsen, an emperor of Tubo during 755-797 CE, strongly supported the development of Buddhism in Tubo and suppressed the Bon religion. The practitioners of the Bon region fled to marginal areas to survive and maintain their religious beliefs. There are several records indicating that the Bon practitioners fled to the eastern Tibetan Plateau, carrying many definitive Bon scriptures (Wang, 2017). Therefore, the inhabitants of the Ganjia Basin, far from the political center of Tubo, may have continued to practice the Bon religion during this period. At the beginning of the 11th century, the Bon religion underwent a brief revival in Tubo, and the Zuohai temple, a Bon monastery built in $1028 \mathrm{CE}$, was a response. At this time, the Zuohai temple and BJC became the religious center for Bon believers in and around the Ganjia Basin. After 1247 CE, with the support of the Mongol Empire, the Sakya sect of Tibetan Buddhism gained the political and religious leadership of the Tubo Kingdom (Chen and Chen, 2015). SRGC, built around 1270 CE, represents the primary spread of Tibetan Buddhism in the region. Subsequently, Tibetan Buddhism gradually replaced the Bon religion and it continues to maintain its status in the 
region. Religions, as specific cultural manifestations and represented by temple factions, also indirectly reflect the prosperity of the Ganjia region during the historical period.

\subsection{History of the integration of the Han and Tibetan peoples as reflected in the Gan- jia Basin}

The Ganjia Basin, as the regional military, economic and religious center for the Plateau ethnic groups during the Tang and Song dynasties, has a unique geographical location and well-preserved ancient cities represented by BJC. It was also a major routeway for military activity and economic and cultural exchange between Gansu and Qinghai provinces during the historical period. The architectural style and unearthed archaeological remains in BJC and SRGC reveal major influences by the cultures of both the Central Plains and the Tibetan Plateau, and in turn the history of these ancient cities also reflects cultural and economic communications between different ethnic groups during the period in which they flourished. Wars also promoted the integration of the ancestral Han and Tibetan peoples to a certain degree. The expansion of the Tubo army represented not only military conquest, but also a process of national migration and integration (Shi, 2014). The Tubo Kingdom carried out a series of political, economic and religious policies which resulted in the assimilation of populations within the newly-conquered regions (Yang, 2010). In addition, the motifs of the Bon religion in BJC convincingly demonstrate that the Tubo Kingdom carried out a policy of assimilation in the Ganjia area. Therefore, war was an important means of promoting communication, exchange and integration between the Tang and Tubo (Wang, 2006). As a military frontline, the history of the repair of the walls of BJC attests to the intensity and frequency of wars and thus reflects the changing relationship between the Tang and Tibet. The then prisoners and plundered populations (Yue, 2008) of the wars also promoted population mobility and cultural exchange between the ancestral Han and Tibetan peoples. In addition, the areas successively occupied and reoccupied by the Central Plains and Plateau regimes also promoted the mixing and blending of the two groups, resulting in closer blood relationships. For example, the Ganjia area was successively occupied by the Tuyuhun, Tubo and Song dynasties, when different peoples, cultures and economic systems merged and co-evolved under the rule of different regimes.

The Tibetan Plateau, with an average elevation above $4000 \mathrm{~m}$ and an area exceeding 2.5 million $\mathrm{km}^{2}$, is a natural barrier which hindered the spread of humans and restricted communication between high-altitude and low-altitude populations. The Central Plains, dominated by the ancestral Han, had little communication with the Qiang people on the Plateau prior to the Tang Dynasty (Ouyang et al., 1975). When Songtsen Gampo established the Tubo Kingdom and dispatched envoys to the Tang Dynasty, the ancestors of the Han and Tibetans, who shared the same origin, began closer contacts. In the early Tang Dynasty, Princess Wencheng married Songtsen Gampo and introduced Chinese culture, advanced production technology, the Buddhist scriptures and the golden figure of the Buddha to the Tubo Kingdom, promoting the development of local agriculture, handicrafts, manufacturing industry, and Tibetan Buddhism (Gazang Caidan, 2013). These developments helped finalize the establishment of the ancient Tang-Bo road on the previous Silk Road and Tea-Horse Road, greatly promoting economic and cultural exchanges between the ancestors of the Han and Tibetans (Cui, 2007). According to statistics, the envoys of Tang and Tubo made at least 
191 exchanges during 634-846 CE (Ma, 2000). In addition, the communication represented by individual trading, marriage and trading amongst peoples became more frequent. In the 11th century, the government of the Northern Song Dynasty supported the Tsongkha, while the Tsongkha also guaranteed the safety of the commercial transportation links from the Northern Song Dynasty to the Western Regions (Liu, 2005). These mutually beneficial exchanges also strengthened the integration of the ancestral Tibetans and the Han in the Gansu and Qinghai regions. In addition, the wars between Tang and Tubo occurred not only in the Longyou area, but also in the Hexi area, the Western Regions, Nanzhao and elsewhere. Therefore, the integration of peoples and cultures in the Ganjia Basin during the historical period was only part of a larger-scale interaction between the Plateau and the Central Plains in their boundary areas, reflecting the history of the interaction of the ancestral Han and Tibetans. The cultural identity and blood relationship produced in the process of this integration played an important role in the establishment of the modern Chinese nation.

\section{Conclusions and prospects}

Based on radiocarbon dating, Bayesian age modeling and historical documents we conclude that $\mathrm{BJC}$, as an important military stronghold, was built during $663-732 \mathrm{CE}$ by the Tubo Kingdom. The early occupation history revealed by the frequency distribution of the radiocarbon ages can be divided into an initial establishment period (670-780 CE) and a regeneration period (880-1030 CE), reflecting the history of frequent warfare in the border area of the Ganjia Basin between the Plateau ethnic groups and the Central Plains peoples. In addition, SRGC was built in $1271 \pm 14$ CE. Combined with its architectural style, we assume that SRGC was a city with a Tibetan Buddhist temple as the center. Combined with historical documentary evidence and previous studies, we have demonstrated the important economic, military and religious status of the Ganjia Basin and the fact that it played an important role in the eastward expansion of the Tubo and in cultural exchange and population movements between and the integration of the ancestral Han and Tibetan peoples. However, our understanding of the history of human activity in the Ganjia Basin remains limited and future more detailed studies of the ancient cities and terraces of the region are likely to provide additional valuable information.

\section{Acknowledgements}

We thank Professor DONG Guanghui of Lanzhou University for his guidance and assistance in research design and paper writing; Director ZHANG Yun of the Institute for Historical Studies, China Tibetology Research Center, for his guidance and assistance with the initial writing of the paper; Dr. LI Hu of Henan Normal University for the identification of the charcoal and wood samples; and the members of the local government of Xiahe County and Ganjia town for their support during fieldwork.

\section{References}

An Jiesheng, 2007. Historical National Geography. Jinan: Shandong Education Press. (in Chinese)

Cai Rangtai, 2006. The preliminary biography of Bon religion in Tubo and its relation with Buddhism. China 
Tibetology, (2): 237-244. (in Chinese)

Chen Fahu, Dong Guanghui, Zhang Dongju et al., 2015. Agriculture facilitated permanent human occupation of the Tibetan Plateau after 3600 BP. Science, 347(6219): 248-250.

Chen Fahu, Welker Frido, Shen Chuanchou et al., 2019. A late Middle Pleistocene Denisovan mandible from the Tibetan Plateau. Nature, 569(7756): 409-412.

Chen Fahu, Xu Qinghai, Chen Jianhui et al., 2015 East Asian summer monsoon precipitation variability since the Last Deglaciation. Scientific Reports, 5: 11186.

Chen Qingying, Chen Lihua, 2015. As the Yuan Dynasty Tibetan Buddhism Sakya. Journal of Qinghai Nationalities University, (3): 11-16. (in Chinese)

Cui Mingde, Ma Xiaoli, 2009. On Tubo's idea of ethnic relations: Taking the relation between Tubo and the Tang Dynasty for example. Journal of Literature, History and Philosophy, (4): 110-118. (in Chinese)

Cui Yonghong, 2007. Princess Wencheng and Tang-Tibet Ancient Road. Xining: Qinghai People's Publishing House. (in Chinese)

Dong Guanghui, Liu Honggao, Yang Yishi, 2016. Emergence of ancient cities in relation to geopolitical circumstances and climate change during late Holocene in northeastern Tibetan Plateau, China. Frontiers of Earth Science, 10(4): 669-682.

Dong Guanghui, Wang Zongli, Ren Lele et al., 2014. A comparative study of ${ }^{14} \mathrm{C}$ dating on charcoal and charred seeds from Late Neolithic and Bronze Age sites in Gansu and Qinghai Provinces, NW China. Radiocarbon, 56(1): 157-163.

Fei Xiaotong, 1999. The Pattern of Diversity in Unity of the Chinese Nation. Beijing: Central University for Nationalities Press. (in Chinese)

Gazang Caidan, 2013. Further discussion on stimulation and continuation of the spirit of Princess Wencheng entering Tibet and cultural exchanges between Chinese and Tibetan. Journal of Tibet University, 28(4): 72-78. (in Chinese)

Ge Quansheng, Liu Jian, Fang Xiuqi et al., 2013. General characteristics of temperature change and centennial warm periods during the past 2000 years. Acta Geographica Sinica, 68(5): 579-592. (in Chinese)

Guo Qinglin, Lu Chun, Liu Ruiliang et al., 2018. New progress in the chronological study of Buddhist Caves: Combining Bayesian modeling with archaeological information to improve dating accuracy. Dunhuang Research, 172(6): 168-176. (in Chinese)

Guo Xiaoming, 2010. Xiahe Cultural Relics Records. Gannan: Xiahe Cultural Relics Records Compilation Committee. (in Chinese)

Jiucaoxi, 2010. Research on the history of Bajiao ancient city in Amdo Ganjia [D]. Beijing: Central University for Nationalities. (in Chinese)

Li Yuchun, Tian Jiaoyang, Liu Fenweng et al., 2019. Neolithic millet farmers contributed to the permanent settlement of the Tibetan Plateau by adopting barley agriculture. National Science Review, 6(5): 1005-1013.

Li Zhenyi, 1994. Survey on the Sirou city site in Ganjia Township of Xiahe County. Journal of Northwest University for Nationalities, (3): 66-69. (in Chinese)

Li Zhenyi, 2012. Investigation of Ancient Cities in Gannan Tibetan Area. Lanzhou: Gansu Ethnic Press. (in Chinese)

Liu Fengwen, Li Haiming, Cui Yifu et al., 2019. Chronology and plant utilization from the earliest walled settlement in the Hexi Corridor, Northwestern China. Radiocarbon, 61(4): 971-989.

Liu Lin, Diao Zhongmin, Shu Dagang et al., 2014. Song Huiyao Jigao. Shanghai: Classics Publishing House. (in Chinese) Liu Xu, 1975. Old Book of Tang. Beijing: Zhonghua Book Company. (in Chinese)

Liu Yanxia, 2005. The roles of Jiao Siluo authority played in the Sino-Foreign trade in eleventh century. Tibetan Studies, (1): 10-12. (in Chinese)

Ma Dazheng, 2000. A Brief History of Chinese Frontier. Zhengzhou: Zhongzhou Ancient Books Publishing House, 2000. (in Chinese)

Nie Chuanping, Hou Yongjian, 2015. Caizaowu, Baozhai, Gongjianshou: The developing of and expanding into the Tubo residential land by the North Song Dynasty. China's Borderland History and Geography Studies, 25(1): 56-64. (in Chinese)

Nowak Marek, Hoyo Magdalena Moskal-del, Mueller-Bieniek Aldona et al., 2017. Benefits and weaknesses of 
radiocarbon dating of plant material as reflected by Neolithic archaeological sites from Poland, Slovakia and Hungary. Geochronometria, 44(1): 188-201.

Ouyang Xiu, Song Qi, Fan Zhen et al., 1975. New Book of Tang. Beijing: Zhonghua Book Company. (in Chinese) Pu Zhongyuan, 2017. Textual research on the Tuyuhun's surrender to the court in the earlier Tang Dynasty. Journal of Hexi University, (6): 41-45. (in Chinese)

Reimer Paula J, Bard Edouard, Bayliss Alex et al., 2013. IntCal13 and Marine13 radiocarbon age calibration curves 0-50,000 years cal BP. Radiocarbon, 55(4): 1869-1887.

Schiffer Michael Brian, 1986. Radiocarbon dating and the "Old Wood" problem: The case of the Hohokam Chronology. Journal of Archaeological Science, 13(1): 13-30.

Shi Shuo, 2014. The ethnic flow of the Tibetan-Yi Corridor in history. Ethno-National Studies, (1): 78-89. (in Chinese)

Shi Yue, 2016. Economic contacts between Tang and Tubo. Journal of Northwest University for Nationalities, (6): 89-95. (in Chinese)

Sima Guang, 2016. Comprehensive Mirror for Aid in Government. Beijing: Joint Publishing Company. (in Chinese)

Tan Qixiang, 1982. The Historical Atlas of China. Beijing: SinoMaps Press. (in Chinese)

Tang Qinfu, Li Risheng, 2017. Some examples of corrections to records of the Liang Dynasty in Zizhitongjian. Journal of Historiography, (1): 104-112. (in Chinese)

Tian Haiying, 2016. Three-dimensional intercrossing: Research on the colonizing process of Tubo dynasty in Gaer family, monopoly period. Journal of Inner Mongolia Normal University (Philosophy \& Social Science), 45(2): 56-61. (in Chinese)

Toqto, 2012. History of Song. Beijing: Zhonghua Book Company. (in Chinese)

Wang Baisui, 2006. A latest study on the war between Tang Dynasty and Tibetan regime: And discussing their relationship [D]. Lanzhou: Lanzhou University. (in Chinese)

Wang Yu, 2017. Investigation on the origin of ancient Tibetan Bon literature of Tanchang region. Tibetan Studies, (5): 47-54. (in Chinese)

Wei Mingkong, 2013. About Tibetan animal husbandry and agriculture in Tang Dynasty: Mainly based on Dunhuang documents. Researches in Chinese Economic History, (2): 47-53. (in Chinese)

Yang Ming, 2010. Becoming Tubo of the ethnic groups in the northwestern Tang Dynasty and its historical effect. Ethno-National Studies, (4): 75-83. (in Chinese)

Yi Ye, 1994. Discrimination on the Yigong city site. Journal of the Northwest Normal University (Social Sciences), (1): 103. (in Chinese)

Yishi Wangmo, Dorji Tserin, 2015. A study on the classification of early Tibetan documents found in the mountain area in south Gansu Province about the Bon religion. Journal of Tibet University, 30(4): 116-123. (in Chinese)

Yizhaq Meirav, Mintz Genia, Cohen Illit et al., 2005. Quality controlled radiocarbon dating of bones and charcoal from the early Pre-Pottery Neolithic B (PPNB) of Motza (Israel). Radiocarbon, 47(2): 193-206.

Yue Xiaoguo, 2008. The war and culture propagation between Tang and Tibet. Journal of Xiaogan University, 28(2): 24-27. (in Chinese)

Zhang Menghan, Yan Shi, Pan Wuyun et al., 2019. Phylogenetic evidence for Sino-Tibetan origin in northern China in the Late Neolithic. Nature, 569(7754): 112-115.

Zhang Pingzhong, Cheng Hai, Edwards R Lawrence et al., 2008. A test of climate, sun, and culture relationships from an 1810-year Chinese cave record. Science, 322(5903): 940-942.

Zheng Jingyun, Liu Yang, Wu Maowei et al., 2019. Evidences and regional differences on multi-scales in Medieval Climate Anomaly over China. Acta Geographica Sinica, 74(7): 1281-1291. (in Chinese)

Zhou Ta, Fan Qiuli, 2010. The textual research on "Yongzhongkaer" city site of Gusiluo. China Tibetology, (1): 77-83. (in Chinese)

Zilhão João, 2001. Radiocarbon evidence for maritime pioneer colonization at the origins of farming in West Mediterranean Europe. Proceedings of the National Academy of Sciences, 98(24): 14180-14185. 